\title{
Simulations and design of detectors for imaging with epithermal neutrons
}

\author{
E Perelli Cippo ${ }^{1}$, G Gorini ${ }^{1}$, M Tardocchi ${ }^{1}$, R Cattaneo ${ }^{1}$, \\ N J Rhodes ${ }^{2}$, E M Schooneveld ${ }^{2}$, T Nakamura ${ }^{2}$, P Radaelli ${ }^{2}$, \\ W A Kockelmann ${ }^{2}$, A Pietropaolo ${ }^{3}$ and the ANCIENT CHARM \\ Collaboration \\ ${ }^{1}$ Dipartimento di Fisica 'Giuseppe Occhialini', Università degli Studi di Milano-Bicocca and \\ CNR-INFM, Piazza della Scienza 3, 20126 Milano, Italy \\ ${ }^{2}$ ISIS Spallation Neutron Source, Rutherford Appleton Laboratory, Chilton, Didcot, \\ OX11 0QX, UK \\ ${ }^{3}$ Dipartimento di Fisica, Università degli Studi di Roma-Tor Vergata and CNR-INFM, Roma, Italy
}

Received 17 July 2007, in final form 13 October 2007

Published 30 January 2008

Online at stacks.iop.org/MST/19/034027

\begin{abstract}
A position sensitive detector for epithermal neutrons is being developed as a tool for neutron resonance tomography at pulsed neutron sources. Neutron-scattering effects in the detector elements were investigated with GEANT4 simulations. The simulations show that the effect of neutron scattering is generally low, and can be further suppressed by proper choice of construction materials.
\end{abstract}

Keywords: neutron imaging detectors, simulations

\section{Introduction}

Ancient Charm is a project financed by the European Community with the aim of developing neutron-based analysis techniques into non-invasive methods for $3 \mathrm{D}$ imaging of the isotopic and phase composition of cultural heritage objects [1]. Among others, the project aims to develop the well-established neutron resonance capture analysis [2-4] into an imaging technique called neutron resonance capture imaging combined with neutron resonance transmission (NRCI/NRT). This is a tomographic method in which both (neutron-induced) gamma emission and neutron transmission of resonant epithermal neutrons will be measured to obtain 3D maps of elemental distributions. The use of a position sensitive neutron detector (PSND) optimized for neutron imaging in the epithermal range $\left(E_{\mathrm{n}}\right.$ up to $1000 \mathrm{eV}$ ) is a key element for NRCI/NRT. The PSND, which is being developed at the ISIS spallation neutron source [5], features a pixelated detector, with the pixels made of neutron-sensitive scintillators. The development of a PSND optimized for epithermal neutrons constitutes a challenge, due mostly to the need of coupling a reasonable detection efficiency for epithermal neutrons to the smallest possible dimensions of the pixels in order to achieve good spatial resolution. A previous PSND developed at ISIS for thermal neutrons had shown good resolution achieved with $2 \times 2 \times 2 \mathrm{~mm}^{3} \mathrm{Li}$-glass scintillator pixels coupled to photomultiplier tubes via optical fibres [6, 7]. For the PSND developed for the Ancient Charm project the depth of a pixel has been extended up to $8 \mathrm{~mm}$ to improve efficiency at neutron energies from the $\mathrm{eV}$ range up to a few keV. Figure 1 shows a comparison between the estimated neutron capture efficiency over the energy band of interest for a $2 \mathrm{~mm}$ and $8 \mathrm{~mm}$ thickness. Although based on the simple exponential attenuation model [8] it suggests an enhancement of neutron capture efficiency of about a factor 3 at an energy of $50 \mathrm{eV}$.

The coupling of neutron-sensitive scintillators with optical fibres and with a frame is normally non-critical for detectors to be used with thermal neutrons. On the other hand, for high neutron energies the scattering effects in the material surrounding the pixels become more relevant. The minimization of the cross-talk between pixels due to the scattering in various detector elements is thus an important design issue. A study of such effects and following optimization of the detector design have been performed with the GEANT4 code [9].

\section{Simulation model}

The simulations were performed in order to evaluate the role of the fibres and of the detector frame in the overall scattering. 


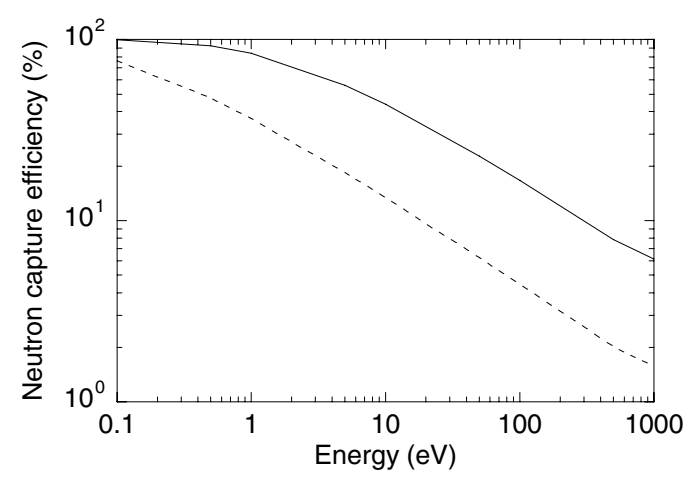

Figure 1. Estimated neutron capture efficiencies for GS20 Li-glass pixels of $8 \mathrm{~mm}$ (full line) and $2 \mathrm{~mm}$ (dashed line) thickness.

Table 1. Model detector configuration. The last column gives the calculated cross-talk levels (see the text).

\begin{tabular}{lllll}
\hline Configuration & Support & Gd paint & Fibres & $\kappa(\%)$ \\
\hline 1 & Al & Y & - & 12.5 \\
2 & Al & Y & Quartz & 19.4 \\
3 & Al & Y & Plastic & 24.8 \\
4 & Al & N & Plastic & 28.9 \\
5 & BN & N & Plastic & 18.3 \\
6 & BN & N & Quartz & 18.2 \\
\hline
\end{tabular}

The GEANT4 model used for the simulations was composed of $10 \times 10 \mathrm{GS} 20 \mathrm{Li}$-glass pixels $\left(6.6 \%\right.$ lithium, with $\left.95 \%{ }^{6} \mathrm{Li}\right)$, embedded in a support frame made of either aluminium or boron nitride (see a sketch of the detector model in figure 2). The dimensions of the pixels are $2 \times 2 \times 8 \mathrm{~mm}^{3}$, with a pitch between adjacent pixels of $2.5 \mathrm{~mm}$ and a frame depth of $16 \mathrm{~mm}$. Each pixel is coupled, via a $5 \mathrm{~mm}$ thick glass disperser, to a small bundle of four $1 \mathrm{~mm}$ diameter fibre optics, composed either of acrylic plastic or quartz. In some of the simulations, a gadolinium oxide neutron absorbing paint was applied to all surfaces of the frame. This was modelled as a $50 \mu \mathrm{m}$ layer of pure gadolinium oxide $\left(\mathrm{Gd}_{2} \mathrm{O}_{3}\right)$, with a density correction factor accounting for the ligant and solvent of the paint. The fibres outside the support frame are collected in a bundle, and they are bent upwards with a bending radius of $10 \mathrm{~cm}$, in order to remove them from the primary neutron beam. The various investigated combinations of frame and fibre materials are shown in table 1. For each detector configuration, the simulation input was a neutron beam of uniform energy distribution between 50 and $100 \mathrm{eV}$, perpendicular to the detector surface. The incident neutron positions were randomly selected inside a $2.5 \times 2.5 \mathrm{~mm}^{2}$ square containing a reference pixel chosen to be close to the centre of the array, namely pixel No. 65 (i.e., the pixel in the 6th row and 5th column). The neutrons were allowed to propagate into the detector volume. A fraction of the neutrons is absorbed in one of the detector pixels.

The output of the simulations was the energy spectrum of recorded events in each pixel. In general, the spectra were always composed of a main component due to the neutron capture in ${ }^{6} \mathrm{Li}$ and a much weaker component of events of

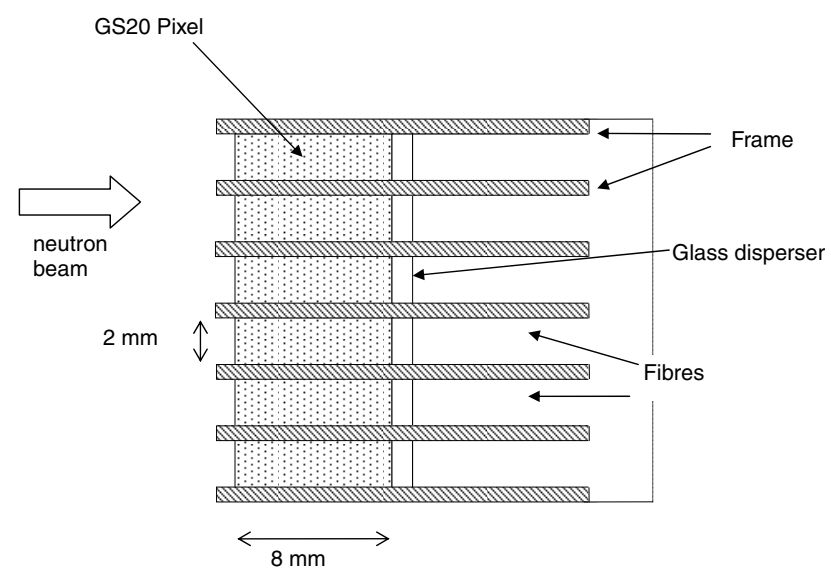

Figure 2. Sketch of the PSND model used in the simulations.

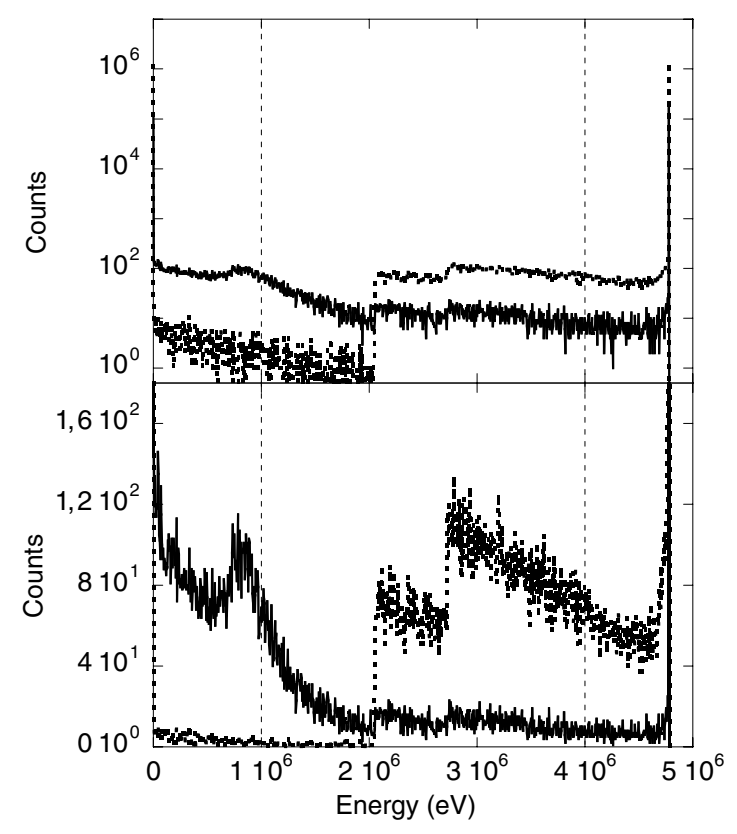

Figure 3. Energy spectrum of the events detected in pixel 65 (dashed line) and in all other pixels (full line) for the model simulation with configuration No. 1 (see text). Top: log scale; bottom: linear scale. In the linear scale the full peak is out of range. The dashed vertical lines are the LLD thresholds used for quantification of cross-talk.

lower energy. The latter component proved to be configuration dependent (see below).

\section{Neutron scattering in the detector}

\subsection{Cross-talk between pixels}

In figure 3 results from the simulation with configuration No. 1 are presented. The configuration No. 1 includes an aluminium frame coated with gadolinium oxide paint and no optical fibres. The figure shows the energy spectrum of the events detected in pixel 65 (dashed line) and the sum of the events detected in all the other pixels (full line). Most of the signal in pixel 65 is 
due to complete energy deposition of the secondary particles from the neutron capture in lithium:

$$
{ }_{3}^{6} \mathrm{Li}+{ }_{0}^{1} \mathrm{n} \rightarrow{ }_{1}^{3} \mathrm{H}+{ }_{2}^{4} \alpha \frac{Q \text {-value }}{4.78 \mathrm{MeV}},
$$

which results in a full energy peak at an energy corresponding to the reaction $Q$-value. We have to note that the simulations only account for the energy deposition in the scintillator pixel, so that the simulated response function does not present any broadening due to, for instance, incomplete light collection in the photomultiplier tube. Other neutron absorption events are detected at lower energies, due to the escape of one of the secondary particles, giving rise to the so-called 'wall effect' [8].

In order to determine a suitable value of the cross-talk level, only events above a suitable low level discrimination (LLD) threshold are considered. Since the expected energy resolution of the GS20 scintillators is about $10 \%$ in the energy range of interest, the threshold is set to about $90 \%$ of the full peak energy, i.e. to $4 \mathrm{MeV}$.

The cross-talk level can be defined as

$$
\kappa^{\prime}=\frac{\sum_{4 \mathrm{MeV}}^{\infty} \text { counts in any other pixel }}{\sum_{4 \mathrm{MeV}}^{\infty} \text { counts in pixel } 65} .
$$

On the other hand, the spectrum recorded elsewhere (i.e., in pixels other than 65) shows a dramatic increase in the low energy part, due to the gamma emission from ${ }^{157} \mathrm{Gd}$ after neutron capture. We have to note that the light output in glass scintillators for heavy charged particles is lower than for gamma rays by about a factor of 4 [8]. As a consequence, the LLD fixed at a specific voltage value would act differently on analogue pulses produced by photons and neutrons. Thus, a realistic cross-talk value should take into account counts of energy higher than $1 \mathrm{MeV}$ in case they are produced by photons. Since at this level of the simulation we do not distinguish between gamma rays and neutrons, we can have a conservative (upper limit) estimation of $\kappa$ defined as the following

$$
\kappa=\frac{\sum_{1 \mathrm{MeV}}^{\infty} \text { counts in any other pixel }}{\sum_{4 \mathrm{MeV}}^{\infty} \text { counts in pixel } 65} .
$$

With this definition we obtain $\kappa=12.5 \%$ for configuration No. 1. The cross-talk levels as defined in equation (2) are given in table 1 for the different investigated detector configurations. These actually present overestimations of the cross-talk level, since not all the events detected in the other pixels (the full plot in figure 3) are due to photons.

The comparison of cross-talk for different configurations in table 1 shows that optical fibres can have a significant scattering effect, in particular if acrylic plastic fibres are used which have a high content of hydrogen. The presence of plastic fibre optics accounts for an increase of at least $12 \%$ of the overall cross-talk level. The use of quartz fibres slightly reduces this value, as does the coating with gadolinium oxide paint.

A significant reduction of the cross-talk can be obtained replacing the aluminium frame with one made of boron nitride. This material presents good mechanical characteristics for the purpose and it is available in sheets of the required thickness

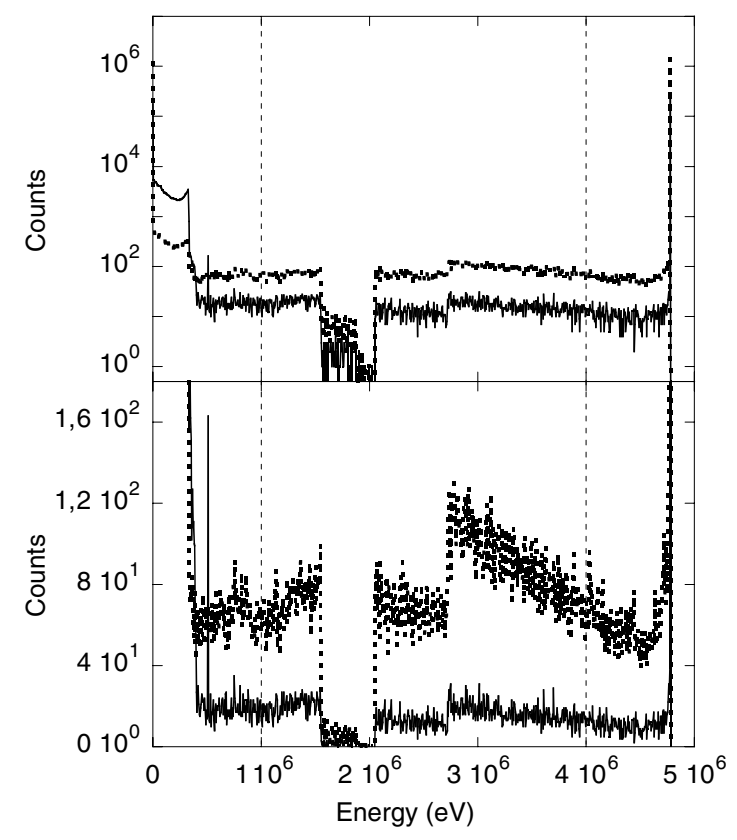

Figure 4. Same as figure 3 but for configuration No. 5. The edges in the signal visible at about $1500 \mathrm{keV}$ are due to the 'wall effect' in the adjacent pixels (see the text for details). The peak at $480 \mathrm{keV}$ is the ${ }^{10} \mathrm{~B}$ emission line after neutron capture.

$(0.5 \mathrm{~mm})$. The ${ }^{10} \mathrm{~B}$ isotope strongly absorbs neutrons via the reaction

$$
{ }_{5}^{10} \mathrm{~B}+{ }_{0}^{1} \mathrm{n} \rightarrow\left\{\begin{array}{l}
{ }_{3}^{7} \mathrm{Li}+{ }_{2}^{4} \alpha \\
{ }_{3}^{7} \mathrm{Li}+{ }_{2}^{4} \alpha+h v
\end{array}\right.
$$

where $94 \%$ of all reactions lead to the emission of a characteristic $480 \mathrm{keV}$ photon.

The reason for such a significant reduction can be understood by the inspection of figure 4 , showing the spectra resulting from a simulation with model configuration No. 5 .

The features of this spectrum can be interpreted considering that the capture of neutrons in ${ }^{10} \mathrm{~B}$ produces heavy charged particles that are emitted in opposite directions. Thus, the two edges visible in the spectrum at about $1500 \mathrm{keV}$ are equivalent, for charged particles, to the escape peaks for photons. At $480 \mathrm{keV}$, the characteristic boron gamma line is visible, as well as the related Compton edge. This line is well below the LLD threshold and hence does not contribute to the cross-talk. The total cross-talk events amount to $\kappa=$ $18.3 \%$, which is better than any of the configurations with an aluminium frame (except the idealized configuration without optical fibres).

A map of signals (normalized to the reference pixel) detected in every pixel in this configuration is presented in table 2 . In the ideal case, the signal should be only detected in the reference pixel (5th column, 6th row). In the simulated case, the cross-talk is at a $1 \%$ level or less, even in the adjacent pixels. The resolution of the detector is of course determined by the lateral dimension of the pixels, i.e., $2 \mathrm{~mm}$ : as can be estimated from figure 5, which shows the same data as in the 
Table 2. Signals detected in the 100 pixels with the model of configuration No. 5.

\begin{tabular}{rrrrrrrrrr}
\hline 0.03 & 0.04 & 0.05 & 0.06 & 0.07 & 0.06 & 0.05 & 0.05 & 0.04 & 0.03 \\
0.05 & 0.06 & 0.08 & 0.10 & 0.11 & 0.10 & 0.08 & 0.06 & 0.05 & 0.03 \\
0.06 & 0.09 & 0.13 & 0.16 & 0.19 & 0.16 & 0.13 & 0.09 & 0.06 & 0.04 \\
0.08 & 0.12 & 0.20 & 0.30 & 0.39 & 0.30 & 0.20 & 0.13 & 0.08 & 0.05 \\
0.09 & 0.16 & 0.29 & 0.63 & 1.11 & 0.62 & 0.29 & 0.16 & 0.09 & 0.06 \\
0.11 & 0.19 & 0.38 & 1.08 & $\mathbf{1 0 0 . 0 0}$ & 1.09 & 0.38 & 0.19 & 0.12 & 0.07 \\
0.09 & 0.15 & 0.30 & 0.59 & 1.06 & 0.61 & 0.29 & 0.16 & 0.10 & 0.06 \\
0.08 & 0.13 & 0.20 & 0.29 & 0.38 & 0.29 & 0.19 & 0.13 & 0.08 & 0.05 \\
0.06 & 0.09 & 0.13 & 0.16 & 0.19 & 0.16 & 0.13 & 0.09 & 0.07 & 0.04 \\
0.05 & 0.06 & 0.07 & 0.09 & 0.11 & 0.09 & 0.08 & 0.06 & 0.05 & 0.03 \\
\hline
\end{tabular}

Table 3. Undesired counts detected in a reference pixel with and without a $200 \mathrm{~mm}$ boron nitride collimator.

\begin{tabular}{lll}
\hline & $\begin{array}{l}\text { Total counts in the } \\
\text { reference pixel, no LLD }\end{array}$ & $\begin{array}{l}\text { Total counts in the reference } \\
\text { pixel, 4 MeV LLD }\end{array}$ \\
\hline No collimator, $E_{\mathrm{n}}=500 \mathrm{eV}$ & 1290 & 186 \\
No collimator, $E_{\mathrm{n}}=1000 \mathrm{eV}$ & 1311 & 132 \\
$200 \mathrm{~mm}$ collimator, $E_{\mathrm{n}}=500 \mathrm{eV}$ & 68 & 16 \\
$200 \mathrm{~mm}$ collimator, $E_{\mathrm{n}}=1000 \mathrm{eV}$ & 107 & 20 \\
\hline
\end{tabular}

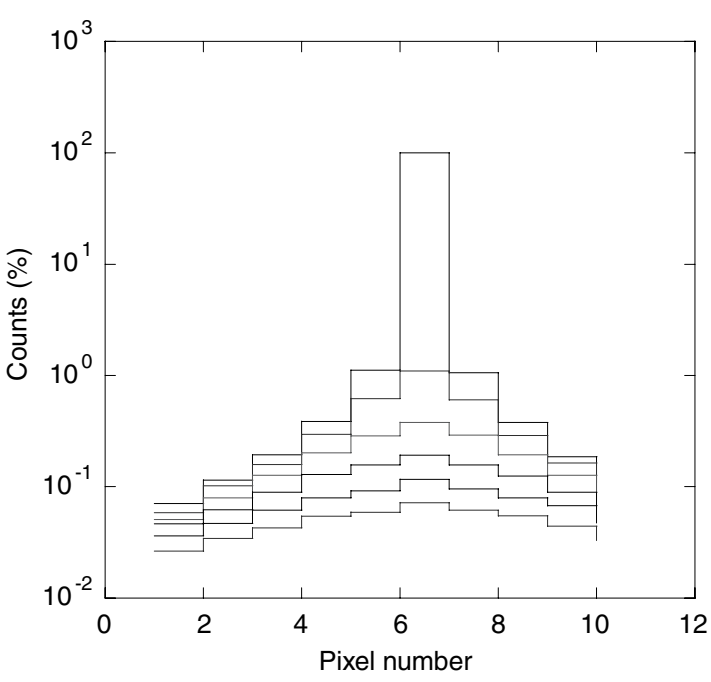

Figure 5. Signal distribution along columns 5-10 of the detector. Column 5 contains the reference pixel No. 65 .

map for columns 5-10, plotted as histograms in log scale, it is not degraded by scattering effects in the adjacent pixels or in the frame.

\subsection{Detector shielding against neutron scattering in the sample}

In the case of neutron transmission measurements with large samples the neutrons can suffer one or more scattering events. As a result, a narrow, parallel beam is widened and becomes slightly divergent. This can cause an effective degradation of the spatial resolution. A suitable collimation system between the sample and the detector itself could reduce the number of neutrons with directions non-parallel with the incoming beam. While the use of so-called 'honeycomb' collimators is known for thermal neutrons (see for instance reference [10]), such a collimator was never considered for epithermal neutrons.
The simplest way to incorporate such a collimator is by extending the boron nitride neutron-absorbing frame for a certain length over the pixel front surface. As an example, the use of a boron nitride collimator of $200 \mathrm{~mm}$ long was compared with the 'standard' detector model presented in figure 2. In order to simulate the effects for a less favourable case, $500 \mathrm{eV}$ and $1000 \mathrm{eV}$ neutrons were used as input emitted from a slab source of dimensions $50 \times 50 \times$ $100 \mathrm{~mm}^{3}$ (representative of the dimensions of a typical Ancient Charm object), excluding the region of the sample just in front of the representative pixel. In other words, such a source would cause only undesired counts in the representative pixel. The neutrons were emitted with a maximum divergence of $12^{\circ}$ in order to illuminate the whole detector. The counts recorded in the reference pixel are summarized in table 3 . Results indicate that the use of a $200 \mathrm{~mm}$ boron nitride neutron collimator in front of the PSND allows for a tenfold reduction of undesired counts at $500 \mathrm{eV}$ and for a sixfold reduction at $1000 \mathrm{eV}$. Therefore, the use of a collimator could be useful for improving the spatial resolution of the detector.

\section{Effect of scattering on the shape of the time-of-flight spectrum of neutron absorption resonances}

The NRCA/NRCI technique requires the accurate reconstruction of time-of-flight (TOF) spectra of the neutrons transmitted through the investigated sample. In particular, much of the information is in the shape and intensity of the 'dips' created by resonant absorption in the sample. Neutron scattering in the detector or in the surrounding components can have the effect of distorting the shape of such dips. In order to isolate the effect of the fibre optics, simulations have been performed with acrylic plastic as well as quartz fibres, and compared with the case with no fibres at all. For the sake of simplicity, we concentrate here on the model with a boron nitride frame, with and without plastic fibres. 


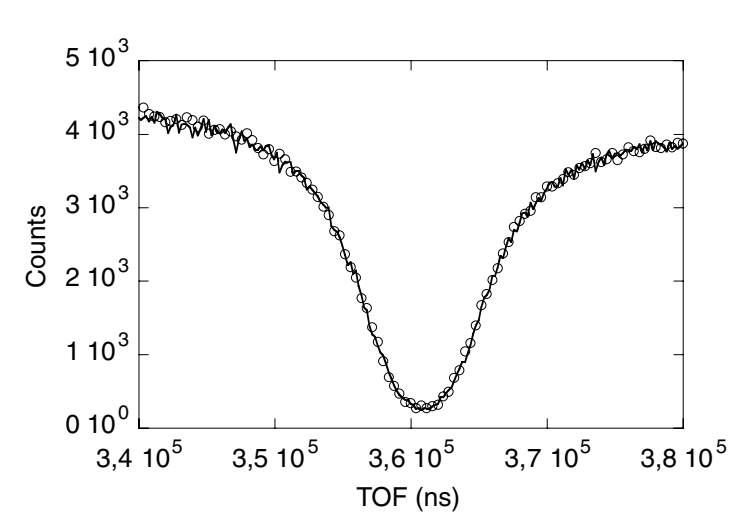

Figure 6. Simulated TOF spectrum in the region of the ${ }^{179} \mathrm{Au}$ absorption resonance at $4.9 \mathrm{eV}$ of the PSND model with acrylic plastic fibre optics (full line) and for the model without fibres (white circles).

Table 4. Input data for three simulations on neutron absorption resonances. The input energy is selected in an energy interval $\Delta E$ around the mean energy resonance value. Red. $\chi^{2}$ is the reduced chi-square calculated from the spectra with and without plastic fibre optics.

\begin{tabular}{lllll}
\hline Run & Foil material & $E_{\mathrm{r}}(\mathrm{eV})$ & $\Delta E(\mathrm{eV})$ & Red. $\chi^{2}$ \\
\hline 1 & ${ }^{179} \mathrm{Au}$ & 62 & $55-70$ & 1.05 \\
2 & ${ }^{238} \mathrm{U}$ & 20 & $10-30$ & 0.93 \\
3 & ${ }^{179} \mathrm{Au}$ & 4.9 & $1-10$ & 0.94 \\
\hline
\end{tabular}

The simulations were performed with a 'white' neutron beam emitted at a distance of $11.055 \mathrm{~m}$ from the detector (the same distance between the sample position and the moderator wall as for the VESUVIO instrument at ISIS). The energy distribution of the neutron beam is proportional to $1 / E^{0.9}$, that is the shape of the under moderated neutron beam on VESUVIO [11]. In order to optimize the running time of the simulations, the energies of the neutrons were limited to an interval around selected absorption resonances. The GEANT4 code allows for the simulation of the neutron resonant capture in selected atomic nuclei, making use of the ENDF/B-VI data library [12]. The input beam was, as before, directed towards the reference pixel, but before striking it, it had to pass through an absorber foil of different thicknesses and materials (gold and uranium) wherein neutrons with energies close to the resonance energies were absorbed. In order to investigate the detector response at different energies, three absorption resonances have been chosen, namely the ${ }^{179} \mathrm{Au}$ resonances at $4.9 \mathrm{eV}$ and at $62 \mathrm{eV}$ and the ${ }^{238} \mathrm{U}$ resonance at $20 \mathrm{eV}$. The thickness of the absorber foil was $50 \mu \mathrm{m}$ except $12.5 \mu \mathrm{m}$ for the $4.9 \mathrm{eV}$ resonance of gold. A reduced thickness was required for the gold foil to avoid 'saturation' effects on the line shape due to the high cross-section values of the $4.9 \mathrm{eV}$ resonance.

The TOF spectrum of the neutrons detected in the reference pixel was recorded, along with the TOF spectra of all the other pixels. The TOF binning of the spectra was assumed to be $200 \mathrm{~ns}$, representative of the binning currently achievable by the ISIS data acquisition electronics. As for previous simulations, a LLD threshold was set to $E=4 \mathrm{MeV}$.
The input data of three simulation runs are summarized in table 4. The results indicate that the distortion of TOF spectra due to the scattering is negligible for all cases, as certified by the reduced $\chi$ value calculated for the two spectra with and without the fibre optics. As an example, figure 6 demonstrates the good agreement between the simulated resonance with plastic fibre optics and without fibres in the region of the $4.9 \mathrm{eV}$ gold absorption resonance.

\section{Conclusions}

GEANT4 simulations have been performed to compare different design configurations of a new position sensitive neutron detector, which is being developed for epithermal neutron transmission measurements in the Ancient Charm project. In particular, the effects of cross talk between different pixels have been studied. The most promising detector configuration features a boron nitride frame and either quartz or plastic fibres, for which the cross-talk level is limited to about $18 \%$. Distortion effects in the TOF shape of neutron absorption resonances caused by scattering effects have been investigated. Results have shown that no visible distortion effects are present for the available data acquisition time binning (200 ns), even for the most critical case (plastic fibres and lower neutron energy). In the case of large samples neutron scattering can degrade the spatial resolution. It has been shown that a suitable neutron collimator in front of the detector reduces significantly the undesired counts due to scattering inside the sample.

\section{Acknowledgments}

This work was performed with financial support by the European Community 'New and Emerging Science and Technology' Contract No. 15311. One of the authors (EPC) acknowledges the Accademia Nazionale dei Lincei for funding. Oliviero Cremonesi and Paolo Dini of University of Milano-Bicocca are gratefully acknowledged for fruitful discussions.

\section{References}

[1] Gorini G and the Ancient Charm Collaboration 2007 Ancient Charm: a research project for neutron-based investigation of cultural-heritage objects Nuovo Cimento C 3047

[2] Postma H, Blaauw M, Bode P, Mutti P, Corvi F and Siegler P 2001 Neutron-resonance capture analysis of materials J. Rad. Nucl. Chem. 248115

[3] Blaauw M, Postma H and Mutti P 2003 Quantitative neutron capture resonance analysis verified with instrumental neutron activation analysis Nucl. Instrum. Methods Phys. Res. A $\mathbf{5 0 5} 508$

[4] Blaauw M, Postma H and Mutti P 2005 An attempt to date an antique Benin bronze using neutron resonance capture analysis Appl. Rad. Isotopes $\mathbf{6 2} 429$

[5] http://www.isis.rl.ac.uk/

[6] Steuwer A, Withers P J, Santisteban J R and Edwards L 2005 Using pulsed neutron transmission for crystalline phase imaging and analysis J. Appl. Phys. 97074903 
[7] Santisteban J R, Edwards L, Fizpatrick M E, Steuwer A and Withers P J 2002 Engineering applications of Bragg-edge neutron transmission Appl. Phys. A 74 (Suppl.) S1433

[8] Knoll G F 2000 Radiation Detection and Measurement 3rd edn (New York: Wiley)

[9] Agostinelli S et al 2003 Geant4-a simulation toolkit Nucl. Instrum. Methods Phys. Res. A 506250

[10] Petrillo C, Guarini E, Formisano F, Sacchetti F, Babucci E and Campeggi C 2002 A honeycomb collimator for the neutron
Brillouin scattering spectrometer BRISP Nucl. Instrum. Methods Phys. Res. A 489304

[11] Taylor A D 1984 SNS moderator performance predictions Rutherford Appleton Laboratory Report RAL-84-120 (Oxford: Rutherford Appleton Laboratory)

[12] Tuli J 1987 Evaluated Nuclear Structure Data File BNL NCS 51655 Rev 87 (New York: Brookhaven National Laboratory) 